\title{
Regulation of Surfactant Phosphatidylcholine Secretion from Alveolar Type II Cells during Pneumocystis carinii Pneumonia in the Rat
}

\author{
Ward R. Rice, * Fannie M. Singleton, ${ }^{*}$ Michael J. Linke, ${ }^{\star}$ and Peter D. Walzer \\ * Divisions of Pulmonary Biology and Neonatology, Department of Pediatrics, Children's Hospital Medical Center, Cincinnati, Ohio \\ 45229-2899; 'Veterans Affairs Medical Center and Division of Infectious Diseases, Department of Internal Medicine, \\ University of Cincinnati College of Medicine, Cincinnati, Ohio 45267-0560
}

\begin{abstract}
We used an immunosuppressed rat model to test the hypothesis that normal mechanisms regulating surfactant phosphatidylcholine synthesis and secretion in alveolar type II cells are aberrant in Pneumocystis carinii pneumonia. Animal groups included: group 1, healthy controls; group 2, immunosuppressed, without pneumocystosis; group 3, immunosuppressed with pneumocystosis; group 4, immunosuppressed with wellestablished pneumocystosis treated with trimethoprim-sulfamethoxazole (TMP-SMX). Type II cells were isolated from rats in each group and compared for $\left[{ }^{3} \mathrm{H}\right]$ choline incorporation into phospholipid and response of the type II cells to secretagogues. Incorporation of $\left[{ }^{3} \mathrm{H}\right]$ choline into phospholipid subclasses exhibited significant differences. Incorporation into phosphatidylcholine fell from $89.3 \pm 2.2 \%$ of total incorporation in group 1 control rats to $79.6 \pm 3.1 \%$ in group 3 rats with $P$. carinii pneumonia, while incorporation into sphingomyelin rose from $5.6 \pm 1.2 \%$ in group 1 animals to $15.2 \pm 2.7 \%$ in group 3 rats. Incorporation of $\left[{ }^{3} \mathbf{H}\right]$ choline into phospholipid subclasses in cells from group 2 and group 4 animals was not different from incorporation for group 1 animals. Type II cells from group 1 and group 2 (immunosuppressed control) rats responded appropriately to the secretagogues ATP, TPA, and terbutaline with a marked increase in surfactant phosphatidylcholine secretion; the effect of ATP was also blocked by the lectin, concanavalin A. In contrast, type II cells from group 3 rats failed to respond to the secretagogues with a significant increase in phospholipid secretion. Although treatment of group 4 rats with TMP-SMX markedly reduced the $\boldsymbol{P}$. carinii organism burden, type II cells from these animals also responded poorly to the secretagogues. The depressed type II cell function described here provides a mechanism for the observed decrease in surfactant phospholipids from bronchoalveolar lavage fluid of experimental animals and patients with $P$. carinii pneumonia. The data also suggest this defect may become irreversible with advanced disease. (J. Clin. Invest. 1993. 92:2778-2782.) Key words: antifungal agents • lung • opportunistic infections • phospholipids • pulmonary disease
\end{abstract}

Address correspondence to Dr. Ward R. Rice, Children's Hospital Medical Center, Division of Pulmonary Biology, TCHRF, Elland \& Bethesda Avenues, Cincinnati, OH 45229-2899.

Received for publication 8 March 1993 and in revised form 18 June 1993.

J. Clin. Invest.

(C) The American Society for Clinical Investigation, Inc.

$0021-9738 / 93 / 12 / 2778 / 05 \$ 2.00$

Volume 92, December 1993, 2778-2782

\section{Introduction}

Pneumocystis carinii pneumonia is a leading cause of morbidity and mortality in immunocompromised individuals. The disease is characterized by progressive respiratory insufficiency resulting from severe lung injury. Animal models have contributed important insight concerning the underlying pathogenic mechanisms in Pneumocystis carinii pneumonia. Rats administered corticosteroids spontaneously develop pneumocystosis with histologic features indistinguishable from those of the disease in humans; ultrastructural studies of these animals have documented the sequential changes that occur within the alveolus (1).

Biochemical and physiological studies of $P$. carinii pneumonia have suggested an important role of the surfactant system in disease pathogenesis (2-12). Abnormalities have been described in surfactant proteins and phospholipids, but the specific factors responsible for these changes and the role played by $P$. carinii have not been elucidated. We hypothesized that normal regulatory mechanisms of the alveolar type II cell are disrupted during pneumocystosis, leading to impaired synthesis and secretion of surfactant phospholipids. To test this hypothesis, we compared the phosphatidylcholine secretory mechanisms of type II cells isolated from normal and immunosuppressed rats with and without $P$. carinii pneumonia.

\section{Methods}

Materials. Materials for the type II cell preparation were obtained from sources noted previously $(13) .\left[{ }^{3} \mathrm{H}\right]$ choline chloride and $\left[{ }^{14} \mathrm{C}\right]-$ dipalmitoyl phosphatidylcholine were purchased from New England Nuclear, Boston, MA. Chloroform and methanol were from Fisher Chemical Company, Fair Lawn, NJ. Biosafe II was from Research Products International, Mount Prospect, IL. Egg yolk phosphatidylcholine, ATP and TPA were obtained from Sigma Chemical Company, St. Louis, MO. Terbutaline was from Ciba Geigy Corp., Summit, NJ. Elastase was obtained from Worthington Biochemical Corp., Freehold, NJ.

Animals. Adult male Sprague-Dawley rats were used in these studies. Group 1 rats, which served as healthy controls, were obtained from Sasco, Inc. (Omaha, NE) or Harlan Industries (Indianapolis, IN); no differences were noted in the function of the type II cells from animals obtained from these vendors. The rats consumed normal food and water ad lib. and received no medications.

Group 2 rats were obtained from a Sasco colony that was free from infection with major rodent viruses and other pathogens, as determined by a regular monitoring program of serology, culture, and pathology (14). These "virus-negative" animals were also free from latent $P$. carinii infection. Group 3 and group 4 rats were obtained from

1. Abbreviations used in this paper: Con A, concanavalin A; SP-A, surfactant-associated protein A; TMP-SMX, trimethoprim-sulfamethoxazole; TPA, 12-O-tetradecanoylphorbol 13-acetate. 
another Sasco colony that was infected with RPO, a parvovirus of low virulence, as determined by the monitoring program (14). These "virus-positive" animals also harbored naturally acquired latent $P$. carinii infection. The virus profile and $P$. carinii infection had been stable in this colony for over a decade.

The animals were housed under barrier conditions and placed on the following immunosuppressive regimen for $10 \mathrm{wk}$ to induce the development of pneumocystosis: methylprednisolone acetate (DepoMedrol; The Upjohn Co., Kalamazoo, MI) 4 mg injected subcutaneously once weekly, a normal diet, and an antibiotic (cephradine or ampicillin, $1 \mathrm{mg} / \mathrm{ml}$ ) in the drinking water $(14,15)$. Group 2 virus-negative or naive rats failed to develop pneumocystosis and thus served as immunosuppressed controls. Groups 3 and 4 virus-positive animals developed active $P$. carinii pneumonia. Group 3 rats received no anti$P$. carinii drugs, whereas group 4 rats were administered trimethoprim (TMP) $150 \mathrm{mg} / \mathrm{kg}$ per d-and-sulfamethoxazole (SMX) $250 \mathrm{mg} / \mathrm{kg}$ per $\mathrm{d}$ orally during the last $3 \mathrm{wk}$ of the immunosuppressive regimen when pneumocystosis had become well established. Sentinel animals were sacrificed periodically throughout the course of immunosuppression to monitor the development of the disease.

Analysis of $P$. carinii infection. At the time of sacrifice, the lungs of each rat were removed and prepared for isolation of type II cells, as described below. This required some modification of our standard procedures for analyzing the extent of $P$. carinii infection. One approach was to stain supernatants of 1-d cultures of type II cells with Diff-Quick (American Scientific Products, McGaw Park, IL), a variant of the Wright-Giemsa stain, to obtain a semiquantitative estimate of the organism burden and to check for the presence of other microbes. The other approach was to sacrifice rats from these rat groups, which were not used for type II cell isolation, and then process their lungs for $P$. carinii quantitation, using procedures reported in detail previously ( 15 , 16). In brief, this involved homogenization of the lungs in a Stomacher (Tekmar, Inc., Cincinnati, $\mathrm{OH}$ ) and staining with cresyl echt violet, which selectively stains $P$. carinii cysts; the lower limit of detection was $1.1 \times 10^{5}$ organisms/lung.

Type II cell preparation. Type II cells were isolated from rat lungs as previously described (13). The animals were anesthetized with sodium pentobarbitone and the lungs perfused via the pulmonary artery, as previously noted (13). Elastase was used to digest the type II cells from the basement membrane, and type II cells were subsequently obtained by the panning method previously described by Dobbs and colleagues (17).

Surfactant phosphatidylcholine secretion. For control experiments, $\sim 1 \times 10^{6}$ cells were placed in each well of a 24 -well plate in culture for $18 \mathrm{~h}$ in the presence of $1 \mu \mathrm{Ci}\left[{ }^{3} \mathrm{H}\right]$ choline. Cells were washed three times with DME containing $40 \mathrm{mM}$ Hepes and $3.0 \mathrm{mg} / \mathrm{ml} \mathrm{BSA},(\mathrm{pH}$ 7.4). Medium $(1.0 \mathrm{ml})$ was then added to each well and cells were incubated for $30 \mathrm{~min}$ at $37^{\circ} \mathrm{C}$. At the end of this time, secretagogues were added and $\left[{ }^{3} \mathrm{H}\right]$ phosphatidylcholine secretion was determined after $3 \mathrm{~h}$. Medium was aspirated and the cells washed with $0.5 \mathrm{ml}$ of fresh medium. These two samples were then combined and centrifuged for $5 \mathrm{~min}$ to pellet loose cells. The supernatant was then removed and the lipid extracted as previously described (13).

Cells remaining in the wells were extracted with methanol and the lipid fraction was obtained as for the medium (13). Lipid samples were dried overnight and the radioactivity was determined with a $\beta$-scintillation counter. The amount of $\left[{ }^{3} \mathrm{H}\right]$ phosphatidylcholine secretion was calculated as the percentage of total $\left[{ }^{3} \mathrm{H}\right]$ phosphatidylcholine present in the medium relative to the amount present in cells and medium. The amount of $\left[{ }^{3} \mathrm{H}\right]$ phosphatidylcholine released following the 30-min preincubation was subtracted from all samples and was generally $2-3 \%$ of the total lipid secreted.

Lactate dehydrogenase activity was determined in each sample to measure cytotoxicity as previously described. None of the agents utilized in these experiments resulted in statistically significant release of lactate dehydrogenase above control levels, which were generally $1-2 \%$ of total cellular lactate dehydrogenase released after a 3-h incubation period.
Analysis of $\left[{ }^{3} \mathrm{H}\right]$ choline incorporation into phospholipid classes. Cells were scraped from the plates in HBSS and lipid extracted from the type II cells as noted above after addition of $50 \mu \mathrm{g}$ each of phosphatidylcholine, lysophosphatidylcholine, and sphingomyelin as carriers. The lipid phase was dried, resuspended in $50 \mu \mathrm{l}$ of chloroform and spotted on silica gel plates (no. 06-600C; Fisher Scientific Co.). The plates were developed with chloroform/methanol/acetic acid/water (60:25:8:4, $\mathrm{vol} / \mathrm{vol}$ ) and spots were visualized with iodine vapor. Individual spots were scraped into scintillation vials and counted as previously described (13). While values for $\left[{ }^{3} \mathrm{H}\right]$ choline incorporation into $\left[{ }^{3} \mathrm{H}\right]-$ phosphatidylcholine are slightly lower compared to those previously reported (13), cells were not scraped from the plates in the prior work, and this methodologic difference may account for the small differences noted in control incorporation of $\left[{ }^{3} \mathrm{H}\right]$ choline into $\left[{ }^{3} \mathrm{H}\right]$ phosphatidylcholine.

Statistical analysis. Data are reported as mean values \pm SEM. Statistical significance between mean values was determined by ANOVA with Newman-Keuls test. $P$ value $<0.05$ was considered statistically significant.

\section{Results}

Incorporation of $\left[{ }^{3} \mathrm{H}\right]$ choline into phospholipid classes differed significantly among the rat groups (Table I). For example, incorporation into phosphatidylcholine fell from $89.3 \pm 2.2 \%$ of total incorporation in type II cells of group 1 control rats to $79.6 \pm 3.1 \%$ in type II cells of group 3 animals with pneumocystosis; this was accompanied by a corresponding rise in the incorporation of $\left[{ }^{3} \mathrm{H}\right]$ choline into sphingomyelin. $\left[{ }^{3} \mathrm{H}\right]$ choline incorporation into sphingomyelin was $5.6 \pm 1.2 \%$ in group 1 animals and rose to $15.2 \pm 2.7 \%$ in group 3 animals with pneumocystosis. Levels of incorporation of $\left[{ }^{3} \mathrm{H}\right]-$ choline into phosphatidylcholine and sphingomyelin in type II cells isolated from group 4 animals were not significantly different from control group 1 or 2 animals, suggesting normalization of $\left[{ }^{3} \mathrm{H}\right]$ choline incorporation in type II cells isolated from animals receiving treatment for pneumocystosis with trimethoprim and sulfamethoxazole. Incorporation of $\left[{ }^{3} \mathrm{H}\right]$ choline into $\left[{ }^{3} \mathrm{H}\right]$ lysophosphatidylcholine was not different in any experimental group.

The phorbol ester, TPA, caused a significant release of surfactant phosphatidylcholine from alveolar type II cells isolated

Table I. $\left[{ }^{3}\right.$ H]Choline Incorporation into Phospholipid Classes for the Experimental Groups

\begin{tabular}{lccrc}
\hline & \multicolumn{4}{c}{ \% of total incorporation* } \\
\cline { 2 - 5 } Rat group & $\begin{array}{c}\text { Phosphatidylcholine } \\
(\text { PC) }\end{array}$ & LysoPC & Sphingomyelin & Other \\
\hline Group 1 & $89.3 \pm 2.2$ & $4.7 \pm 1.7$ & $5.6 \pm 1.2$ & $0.4 \pm 0.1$ \\
Group 2 & $89.6 \pm 1.7$ & $5.0 \pm 1.6$ & $4.9 \pm 0.5$ & $0.5 \pm 0.1$ \\
Group 3 & $79.6 \pm 3.1$ & $4.9 \pm 1.3$ & $15.2 \pm 2.7$ & $0.3 \pm 0.1$ \\
Group 4 & $85.2 \pm 2.5$ & $6.3 \pm 2.2$ & $8.1 \pm 2.4$ & $0.5 \pm 0.2$
\end{tabular}

* Data represent the mean \pm SEM obtained from two random samples of type II cells obtained for each animal, with three to four animals in each group. Samples were determined in duplicate. Incorporation of $\left[{ }^{3} \mathrm{H}\right]$ choline into phosphatidylcholine and sphingomyelin for group 3 (pneumocystosis) animals was significantly different from control (group 1) incorporation ( $P=0.03$ for incorporation into phosphatidylcholine and $P=0.004$ for incorporation into sphingomyelin). 
Table II. $\left[{ }^{3} \mathrm{H}\right]$ Phosphatidylcholine Released from Type II Cells in Response to Secretagogues for the Experimental Groups

\begin{tabular}{cccccc}
\hline & \multicolumn{5}{c}{$\left.\%{ }^{3} \mathrm{H}\right]$ Phosphatidylcholine released } \\
\cline { 2 - 6 } Rat group & Control & ATP & ATP + ConA & TPA & Terbutaline \\
\hline Group 1 & $1.2 \pm 0.3$ & $2.6 \pm 0.6$ & $0.1 \pm 0.2$ & $5.2 \pm 1.5$ & $2.3 \pm 0.8$ \\
Group 2 & $2.9 \pm 0.6$ & $9.4 \pm 1.3$ & $2.0 \pm 0.4$ & $12.0 \pm 2.3$ & $5.9 \pm 0.8$ \\
Group 3 & $1.1 \pm 0.2$ & $1.4 \pm 0.2$ & $0.5 \pm 0.1$ & $1.1 \pm 0.3$ & $1.2 \pm 0.2$ \\
Group 4 & $2.6 \pm 0.6$ & $2.7 \pm 0.5$ & $0.8 \pm 0.2$ & $4.1 \pm 1.1$ & $2.7 \pm 0.3$
\end{tabular}

\begin{abstract}
* Release of $\left[{ }^{3} \mathrm{H}\right]$ phosphatidylcholine was determined after a 3-h incubation as stated in Methods. The concentrations of agents utilized were ATP, $1 \times 10^{-5} \mathrm{M}$; concanavalin A, $1 \times 10^{-5} \mathrm{~g} / \mathrm{ml}$; TPA, $1 \times 10^{-7} \mathrm{M}$; terbutaline, $1 \times 10^{-5} \mathrm{M}$. These concentrations were previously demonstrated to produce a maximal effect $(13,18)$. Data represent the mean \pm SEM for four to eight animals in each group. Each treatment was performed in triplicate for each animal. For group 1 animals, TPA significantly stimulated secretion $(P=0.03)$. For group 2 animals, control secretion was significantly elevated above control secretion for cells from group 1 animals $(P=0.02)$ and ATP and TPA significantly stimulated secretion above the control level for this group. The ATP-induced secretion was also significantly inhibited by ConA $(P=0.005)$. None of the secretagogues had significant effects on secretion in cells from group 3 or group 4 animals. Control secretion for cells from group 3 and group 4 animals was not statistically different for control secretion for cells from group 1 animals.
\end{abstract}

from group 1 (healthy control) rats ( Table II). The lectin, concanavalin A (Con A), also caused significant inhibition of ATP-induced surfactant phosphatidylcholine secretion, as previously described (18). Similar results were observed with type II cells from group 2 rats which lacked latent $P$. carinii infection and served as immunosuppressed controls (Table II). ATP and TPA caused significant augmentation of surfactant phosphatidylcholine secretion relative to the control for group 2, whereas Con A exerted an inhibitory effect. Control secretion in cells from group 2 animals was also significantly elevated above control secretion compared to cells from group 1 animals. $P$. carinii cyst counts performed on seven group 2 rats were all $\leq 1.1 \times 10^{5} /$ lung.

In contrast, alveolar type II cells obtained from group 3 rats, which developed $P$. carinii pneumonia with immunosuppression, failed to show normal regulation of surfactant phosphatidylcholine secretion ( Table II). The potent secretagogues, ATP and TPA, failed to augment phosphatidylcholine secretion in a statistically significant fashion, although control secretion of phosphatidylcholine was similar in cells from group 1 and group 3 animals. $P$. carinii quantitation performed on four group 3 rats revealed a median cyst count of $7.3 \times 10^{7} /$ lung (range $2.2 \times 10^{5}-6.5 \times 10^{8} /$ lung).

Type II cells isolated from group 4 rats, which received $3 \mathrm{wk}$ of treatment with TMP-SMX for their pneumocystosis, showed results similar to those found with type II cells from group 3 rats (Table II). Neither ATP nor TPA significantly increased phosphatidylcholine secretion above the control level for group 4. Also of note, the values obtained for secretagogue-induced secretion from cells obtained from group 4 animals were not different from values obtained for group 1 animals, but were significantly lower than values obtained for control group 2 animals, which were similarly immunosuppressed. $P$. carinii cyst counts performed in seven group 4 animals showed a median count of $\leq 1.1 \times 10^{5} /$ lung (range $\leq 1.1$ $\times 10^{5}-2.2 \times 10^{7} /$ lung). Thus, impaired surfactant secretion from isolated type II cells persisted despite a 700 -fold reduction in median organism burden.

\section{Discussion}

We have demonstrated aberrant regulation of surfactant phosphatidylcholine synthesis and secretion by alveolar type II cells during experimental $P$. carinii pneumonia. Compared with healthy and immunosuppressed control groups, type II cells isolated from rats with pneumocystosis failed to respond to potent secretagogues, ATP and TPA, in a normal fashion. This inhibition persisted after 3 wk of treatment with TMP-SMX and after marked clearing of the infection.

Previous reports have demonstrated that exposure of type II cells to ozone or oxygen radicals resulted in decreased secretion of lamellar bodies and reduced incorporation of surfactant precursors $(19,20)$. We found that type II cells isolated from rats with $P$. carinii pneumonia have decreased incorporation of $\left[{ }^{3} \mathrm{H}\right]$ choline into phosphatidylcholine. The present study also demonstrates the advantage of using an in vitro approach to study type II cell function, since cells obtained from different experimental groups can be compared under similar experimental conditions.

There is increasing clinical and experimental evidence that perturbations in the surfactant system contribute to the respiratory insufficiency in $P$. carinii pneumonia. Reported abnormalities during $P$. carinii pneumonia include impaired gas exchange and altered lung compliance, and administration of surfactant has improved oxygenation and lung architecture (4$6,9)$. Studies of bronchoalveolar lavage fluid have demonstrated a fall in total phosphatidylcholine levels and a rise in sphingomyelin concentrations during $P$. carinii pneumonia ( 5 , $8,10)$. The results of the present study corroborate this work and identify one source of the abnormalities, since most of the bronchoalveolar lavage fluid phospholipid originates in the type II cell and we demonstrated similar findings in the lipid fractions from the isolated type II cells. One group of investigators also found increased levels of phospholipase $A_{2}$, suggesting enhanced catabolism to explain the lower phosphatidylcholine levels observed in bronchoalveolar lavage fluid during pneumocystosis (2). Other workers could not confirm this observation, but did find a difference in lavage phosphatidylcholine levels in experimental groups, suggesting problems with regulation of surfactant secretion during pneumocystosis (5). Data from the present study support the latter work. Not only did we observe impaired regulation of synthesis and secretion of surfactant phosphatidylcholine, but there were no detectable differences among lysophosphatidylcholine levels in the type II cells isolated from the rat groups, arguing against significant degradation of phosphatidylcholine in type II cells. 
Studies of pneumocystosis using animal models require appropriate controls to ensure the biochemical changes observed are not due to components of the immunosuppressive regimen or other organisms present in the airway. We utilized a standard model consisting of rats with naturally acquired latent $P$. carinii infection obtained from a stable colony that is regularly monitored for the presence of major rodent microbial pathogens (14). Alternatively, $P$. carinii infection can be established in naive rats by intratracheal innoculation of infected lung homogenates $(21,22)$. However, this system is less reliable in our laboratory than the alternative model in producing acceptable levels of pneumocystosis. Until it is possible to continuously culture $P$. carinii in vitro, neither approach can unequivocally rule out the presence of fastidious lung microbes which may not be detectable by conventional methods.

One way to directly assess the interaction of $P$. carinii and alveolar type II cells involves the use of isolated $P$. carinii cell wall components. Preliminary work from our laboratory has demonstrated that the isolated major surface glycoprotein of $P$. carinii also inhibits surfactant phospholipid secretion from isolated type II cells in vitro (23), indicating the effects we observed in vivo may indeed be due to presence of $P$. carinii organisms in the airway. However, other factors may also be involved. Studies by Pesanti have suggested a complex interaction of $P$. carinii with type II cells, ranging from a toxic effect of type II cells on the organism to the ability of $P$. carinii to bind surfactant phospholipids $(24,25)$. $P$. carinii also stimulates alveolar macrophages to produce TNF- $\alpha$ (26), which can alter the synthesis of the surfactant associated protein, SP-A (27). Whether TNF- $\alpha$ can also impair phospholipid synthesis or secretion is the subject of current investigation.

In addition to changes in surfactant phospholipids, elevated bronchoalveolar lavage fluid SP-A protein and tissue mRNA levels have been reported in patients and experimental animals with pneumocystosis $(7,11,12)$. This is in contrast to other types of pneumonia, which have been accompanied by a fall in SP-A levels (28). It is unclear whether the augmented expression of SP-A is related to the disordered regulation of phosphatidylcholine secretion described here or is a nonspecific response to lung injury. SP-A has complex interactions with surfactant phospholipids and $P$. carinii (29-35), and thus changes in the level of this protein could have a major impact on the pathophysiology of this disease.

Basal $\left[{ }^{3} \mathrm{H}\right]$ phosphatidylcholine secretion from cells isolated from group 2 immunosuppressed animals was significantly elevated above basal secretion from cells from group 1 animals. The reason for this difference is unknown. Steroids are known to increase expression of surfactant-associated proteins, as well as enzymes involved in synthesis of surfactant phospholipids (36). Whether augmented synthesis of surfactant proteins and phospholipids by treatment with corticosteroids would result in increased basal secretion of phospholipid by type II cells has not been studied, but appears plausible.

The impaired regulatory mechanisms in surfactant phospholipid synthesis and secretion in pneumocystosis presented in this study have important clinical implications. The prognosis of $P$. carinii pneumonia can be related to the degree of respiratory impairment, as expressed by the magnitude of hypoxemia or alveolar-arterial oxygen gradient (37). Treatment with anti- $P$. carinii drugs started early in the course of the disease, when the respiratory dysfunction is mild, is usually more successful than therapy which has been delayed. TMP-
SMX was begun in our rats after 7 wk of immunosuppression when $P$. carinii infection had already reached moderate severity, and did not improve the aberrant phospholipid secretion, although synthesis of phosphatidylcholine was normalized. This raises the possibility that type II cell dysfunction, like other changes in pneumocystosis, becomes irreversible as the disease progresses, and emphasizes the need for early therapeutic intervention.

\section{Acknowledgments}

We thank Jilanna Foy for excellent technical assistance.

This study was supported by the Medical Research Service of the Department of Veterans Affairs and by public service contract AI25139 and grants HIL-46653, AI-31702, and AI-32899 from the National Institutes of Health.

\section{References}

1. Walzer, P. D., C. K. Kim, and M. T. Cushion. 1989. Pneumocystis carinii. In Parasitic Infections in the Compromised Host. P. D. Walzer, and R. M. Genta, editors. Marcel Dekker, New York. 83-178.

2. Kernbaum, S., J. Masliah, L. G. Alcindor, C. Bouton, and D. Christol 1983. Phospholipase activities of bronchoalveolar lavage fluid in rat Pneumocystis carinii pneumonia. Br. J. Exp. Pathol. 64:75-80.

3. Brun-Pascaud, M., J. J. Pocidalo, and S. Kernbaum. 1985. Respiratory and pulmonary alterations in experimental Pneumocystis carinii pneumonia in rats. Bull. Eur. Physiopathol. Resp. 21:37-41.

4. Stokes, D. C., W. T. Hughes, P. O. Alderson, R. E. King, and D. J. Garfinkel. 1986. Lung mechanisms, radiography and ${ }^{67} \mathrm{Ga}$ scintigraphy in experimental Pneumocystis carinii pneumonia. Br. J. Exp. Pathol. 67:383-393.

5. Sheehan, P. M., D. C. Stokes, Y. Yeh, and W. T. Hughes. 1986. Surfactant phospholipids and lavage phospholipase $\mathrm{A}_{2}$ in experimental Pneumocystis carinii pneumonia. Am. Rev. Resp. Dis. 134:526-531.

6. Eijking, E. P., G. J. van Daal, R. Tenbrinck, A. Luijendijk, J. F. Sluiters, E. Hannappel, and B. Lachmann. 1991. Effect of surfactant replacement on Pneumocystis carinii pneumonia in rats. Intensive Care Med. 17:475-478.

7. Phelps, D. S., J. A. Fishman, and R. M. Rose. 1992. Surfactant protein A levels in glucocorticoid-immunosuppressed rats infected with Pneumocystis carinii. Am. Rev. Resp. Dis. 145:A246.

8. Su, T. H., V. Natarajan, and W. J. Martin. 1992. Pulmonary surfactant in Pneumocystis carinii pneumonia is associated with a marked increase in sphingomyelin. Am. Rev. Resp. Dis. 145:A246.

9. Sankary, R. M., J. Turner, A. Lipavsky, E. L. Howes, and J. F. Murray. 1988. Alveolar-Capillary block in patients with AIDS and Pneumocystis carinii pneumonia. Am. Rev. Respir. Dis. 137:443-449.

10. Hoffman, A. G. D., M. G. Lawrence, F. P. Ognibene, A. F. Suffredini, J. A. Kovacs, H. Masur, and J. H. Shelhamer. 1992. Reduction of pulmonary surfactant in patients with human immunodeficiency virus and Pneumocystis carinii pneumonia. Chest. 102:1730-1736.

11. Baughman, R. P., W. Hull, and J. A. Whitsett. 1992. Pneumocystis carinii alters surfactant associated protein-A concentrations found in bronchoalveolar lavage fluid. Clin. Res. 40:412A

12. Phelps, D. S., and R. M. Rose. 1991. Increased recovery of surfactant protein A in AIDS-related pneumonia. Am. Rev. Respir. Dis. 143:1072-1075.

13. Rice, W. R., C. C. Dorn, and F. M. Singleton. 1990. $P_{2}$-purinoceptor regulation of surfactant phosphatidylcholine secretion. Biochemical J. 266:407413.

14. Health Monitoring Report. 1992-1993. Sasco, Inc., Omaha, Nebraska 68101 .

15. Walzer, P. D., J. Foy, P. Steele, C. K. Kim, M. White, R. S. Klein, and B. A. Otter. 1992. Activities of anitfolate, antiviral, and other drugs in an immunosuppressed rat model of Pneumocystis carinii pneumonia. Antibicrob. Agents Chemother. 36:1935-1942.

16. Kim, C. K., J. M. Foy, M. T. Cushion, D. Stanforth, M. J. Linke, H. L. Hendrix, and P. D. Walzer. 1987. Comparison of histologic and quantitative techniques in the evaluation of experimental Pneumocystis carinii. Antimicrob. Agents Chemother. 31:197-201.

17. Dobbs, L. G., R. Gonzalez, and M. C. Williams. 1986. An improved method for isolating Type II cells in high yield and purity. Am. Rev. Respir. Dis. 134:141-145.

18. Rice, W. R., and F. M. Singleton. 1988. Regulation of surfactant phospholipid secretion from isolated rat alveolar Type II cells by lectins. Biochem. Biophys. Acta. 958:205-210. 
19. Haagsman, H. B., E. J. Schuurmans, G. M. Link, J. J. Batenburg, and L. M. G. vanGolde. 1985. Effects of ozone on phospholipid synthesis by alveolar Type II cells isolated from adult rat lung. Exp. Lung Res. 9:67-84.

20. Crim, C., and R. H. Simon. 1988. Effects of oxygen metabolites on rat alveolar Type II cell viability and surfactant metabolism. Lab. Invest. 58:428437.

21. Bartlett, M. S., J. A. Fishman, S. F. Queener, M. M. Durkin, M. A. Jay, and J. W. Smith. 1988. New rat model of Pneumocystis carinii infection. J. Clin. Microbiol. 26:1100-1102.

22. Boylan, C. J., and W. L. Current. 1992. Improved rat model of Pneumocystis carinii pneumonia: induced laboratory infections in Pneumocystis-free animals. Infect. Immun. 60:1589-1597.

23. Rice, W. R., F. M. Singleton, M. J. Linke, and P. D. Walzer. 1993. Control of Type II cell function by Pneumocystis carinii. Am. Rev. Resp. Dis. 147:A35.

24. Pesanti, E. L. 1987. Phospholipid profile of Pneumocystis carinii and its interaction with alveolar type II epithelial cells. Infect. Immun. 55:736-774.

25. Pesanti, E. L. 1991. Interaction of cytokines and alveolar cells with Pneumocystis carinii in vitro. J. Inf. Dis. 163:611-616.

26. Tamburrini, E., A. Deluca, G. Ventura, G. Maiuro, A. Siracusano, E. Ortona, and A. Antinori. 1991. Pneumocystis carinii stimulates in vitro production of tumor necrosis factor- $\alpha$ by human macrophages. Med. Microbiol. Immunol. 180:15-20.

27. Wispé, J. R., J. C. Clark, B. B. Warner, D. Fahardo, W. Hull, R. B. Holtzman, and J. A. Whitsett. 1990. Tumor necrosis factor- $\alpha$ inhibits expression of pulmonary surfactant protein. J. Clin. Invest. 86:1954-1960.

28. Baughman, R. P., R. I. Sternberg, W. Hull, J. Buchsbaum, and J. Whitsett. 1993. Decreased surfactant protein $A$ in patients with bacterial pneumonia. Am. Rev. Resp. Dis. 147:653-657.

29. Dobbs, L. G., J. R. Wright, S. Hawgood, R. Gonzalez, K. Venstrom, and J. Nellenbogen. 1987. Pulmonary surfactant and its components inhibit secretion of phosphatidylcholine from cultured rat alveolar type II cells. Proc. Natl. Acad. Sci. 84:1010-1014.

30. Rice, W. R., G. F. Ross, F. M. Singleton, S. Dingle, and J. A. Whitsett. 1987. Surfactant-associated protein inhibits phospholipid secretion from Type II cells. J. Appl. Physiol. 63:692-698.

31. Wright, J. R., R. E. Wager, S. Hawgood, L. Dobbs, and J. A. Clements. 1987. Surfactant apoprotein $M_{r}=26,000-36,000$ enhances uptake of liposomes by type II cells. J. Biol. Chem. 262:2888-2894.

32. Hawgood, S., B. J. Benson, J. Schilling, D. Damm, J. Clements, and R. T. White. 1987. Nucleotide and amino acid sequences of pulmonary surfactant protein SP 18 and evidence for cooperation between SP 18 and SP 28-36 in surfactant lipid absorption. Proc. Natl. Acad. Sci. 84:66-70.

33. Haagsman, H. P., S. Hawgood, T. Sargeant, D. Buckley, T. White, K. Drickamer, and B. J. Benson. 1987. The major lung surfactant protein, SP 8-36, is a calcium-dependent, carbohydrate-binding protein. J. Biol. Chem. 262:1387713880.

34. Zimmerman, P. E., R. Voelker, F. X. McCormack, J. R. Paulsrud, and W. J. Martin. 1992. 120-kD surface glycoprotein of Pneumocystis carinii is a ligand for surfactant protein A. J. Clin. Invest. 89:143-149.

35. Koziel, H., D. O'Riordan, D. Phelps, J. A. Fishman, M. Y. K. Armstrong, F. F. Richards, and R. M. Rose. 1992. Surfactant protein-A inhibits binding and internalization of Pneumocystis carinii by alveolar machrophages. Am. Rev. Respir. Dis. 145:A247.

36. Ballard, P. L. 1989. Hormonal regulation of pulmonary surfactant. Endocrine Revs. 10:165-181.

37. The National Institutes of Health-University of California Expert Panel for Corticosteroids as Adjunctive Therapy for Pneumocystis carinii Pneumonia. 1990. Consensus statement on the use of corticosteroids as adjunctive therapy for Pneumocystis pneumonia in the acquired immunodeficiency syndrome. $N$. Engl. J. Med. 323:1500-1504 